\title{
Severe Alport Phenotype in a Woman with Two Missense Mutations in the Same COL4A5 Gene and Preponderant Inactivation of the X Chromosome Carrying the Normal Allele
}

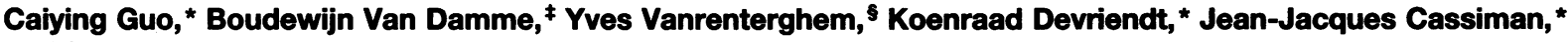 \\ and Peter Marynen* \\ ${ }^{*}$ Human Genome Laboratory, Center for Human Genetics, ${ }^{\ddagger}$ Department of Pathology and ${ }^{\S}$ Department of Nephrology, University \\ of Leuven, Leuven, Belgium
}

\begin{abstract}
The X-linked form of Alport disease, caused by mutations in the COL4A5 or the COL4A6 gene, usually leads to terminal renal failure in males, while affected females have a more variable and moderate phenotype. We detected in a female patient, with a severe Alport phenotype, two new missense mutations. One mutation (G289V) occurred in exon 15 and converted a glycine in a collagenous domain of COL4A5 to a valine. The second mutation, located in exon 46, substituted a cysteine proximal to the NC1 domain of COL4A5 for an arginine. In white blood cells and kidney both mutations were present on $>90 \%$ of the mRNA, while at the genomic level the patient was heterozygous for both mutations. The two mutations therefore occurred in the same COL4A5 allele. No mutation was found in the COLAA5 promoter region by sequencing nor was a major rearrangement of the normal allele detected. A skewed pattern of $\mathrm{X}$ inactivation was demonstrated in DNA isolated from the patient's kidney and white blood cells: $>90 \%$ of the $X$ chromosomes with the normal COL4A5 allele was inactivated. It is suggested that this skewed inactivation pattern is responsible for the absence of detectable normal COL4A5 mRNA and hence the severe phenotype in this woman. ( $J$. Clin. Invest. 1995. 95:1832-1837.) Key words: $\alpha 5$ (IV) collagen chain - COL4A5 mutation • $X$ inactivation - illegitimate transcription
\end{abstract}

\section{Introduction}

Alport disease is characterized by a progressive nephritis and is usually associated with sensorineural deafness and ocular defects. The X-linked form of Alport disease is caused by mutations in the COL4A5 or the COL4A6 gene (1). The disease is more commonly diagnosed in males than females. While the affected males have a more severe phenotype and often progress to terminal renal failure, most of the affected females have moderate clinical features varying from asymptomatic hematuria to renal impairment, though this occurs at an older age than

Address correspondence to Professor P. Marynen, Center for Human Genetics, University of Leuven, Campus Gasthuisberg, Herestraat 49, B-3000 Leuven, Belgium. Phone: 16-34-58-91; FAX: 16-34-59-97; Email: pmarynen@cc3.kuleuven.ac.be.

Received for publication 19 September 1994 and in revised form 5 December 1994.

J. Clin. Invest.

(c) The American Society for Clinical Investigation, Inc. 0021-9738/95/04/1832/06 \$2.00

Volume 95, April 1995, 1832-1837 in men (2). Different mutations of the COL4A5 gene were found in Alport patients: single base substitutions, insertions or deletions, splice mutations, and important rearrangements. These mutations are scattered all over the gene (3). Attempts to correlate mutations with particular phenotypes suggested that mutations resulting in the deletion of the noncollagenous domain would be associated with a more severe phenotype and anti-glomerular basement membrane nephritis after renal transplantation. However, no clear correlation between the disease phenotype and a particular mutation has been reported (4). The variable phenotype in females who are heterozygous for an $\mathrm{X}$ linked disease could be explained by different patterns of $\mathrm{X}$ chromosome inactivation although no correlation between the severity of the disease and the $\mathrm{X}$-inactivation pattern in white blood cells (WBC) ${ }^{1}$ was found (5).

We report two new missense mutations in the COL4A5 gene of a female Alport patient with a severe phenotype. Both mutations were shown to occur on the same COL4A5 gene. The severe Alport phenotype correlated with the observation that only mutant COL4A5 mRNA was detected in kidney tissue. $A$ nonrandom pattern of $\mathrm{X}$ inactivation was demonstrated that could account for the preferential expression of the mutant allele leading to a severe phenotype in this woman.

\section{Methods}

Patient and samples. This female patient presented at the age of 19 with microscopic hematuria and nephrotic syndrome. The diagnosis of Alport disease was confirmed by the finding of typical glomerular basement membrane abnormalities on a renal biopsy taken at the age of 19 . Renal function has deteriorated progressively and chronic hemodialysis was started at the age of 30 . A cadaveric kidney transplantation was done $2 \mathrm{yr}$ later. There was no hearing loss and there were no clinical features of Turner syndrome. Her height was $162 \mathrm{~cm}$ and she had a normal menstruation pattern. Family history revealed that her father died at the age of 36 due to renal failure, associated with sensorineural hearing loss. Her elder sister had microscopic hematuria, proteinuria with normal kidney function, and hearing loss.

Reverse transcription-PCR. RNA was extracted from kidney tissue, WBC, and EBV-transformed cells using the guanidinium thiocyanateacid phenol method. cDNA synthesis, PCR amplification, and sequencing were as described previously (6).

DNA analysis. Genomic DNA was extracted from frozen kidney, WBC, and EBV-transformed cells using a salt-chloroform method (7). For the analysis of exon 15 of COL4A5, a set of primers (F: 5'AACTATTTTTATGTGTACAGGG, R: 5'-TTAGCAGTTACATCACTTACTC) was derived from flanking sequences obtained from $\mathrm{K}$. Tryggvason (University of Oulu, Oulu, Finland). For exon 46, the primers (F:5'-TGCCTCATTCTTTTCCTGTAGGTC, R: 5'-TCCACCAACAGCATGTTTTACTTG) were selected according to the published

1. Abbreviation used in this paper: $\mathrm{WBC}$, white blood cells. 


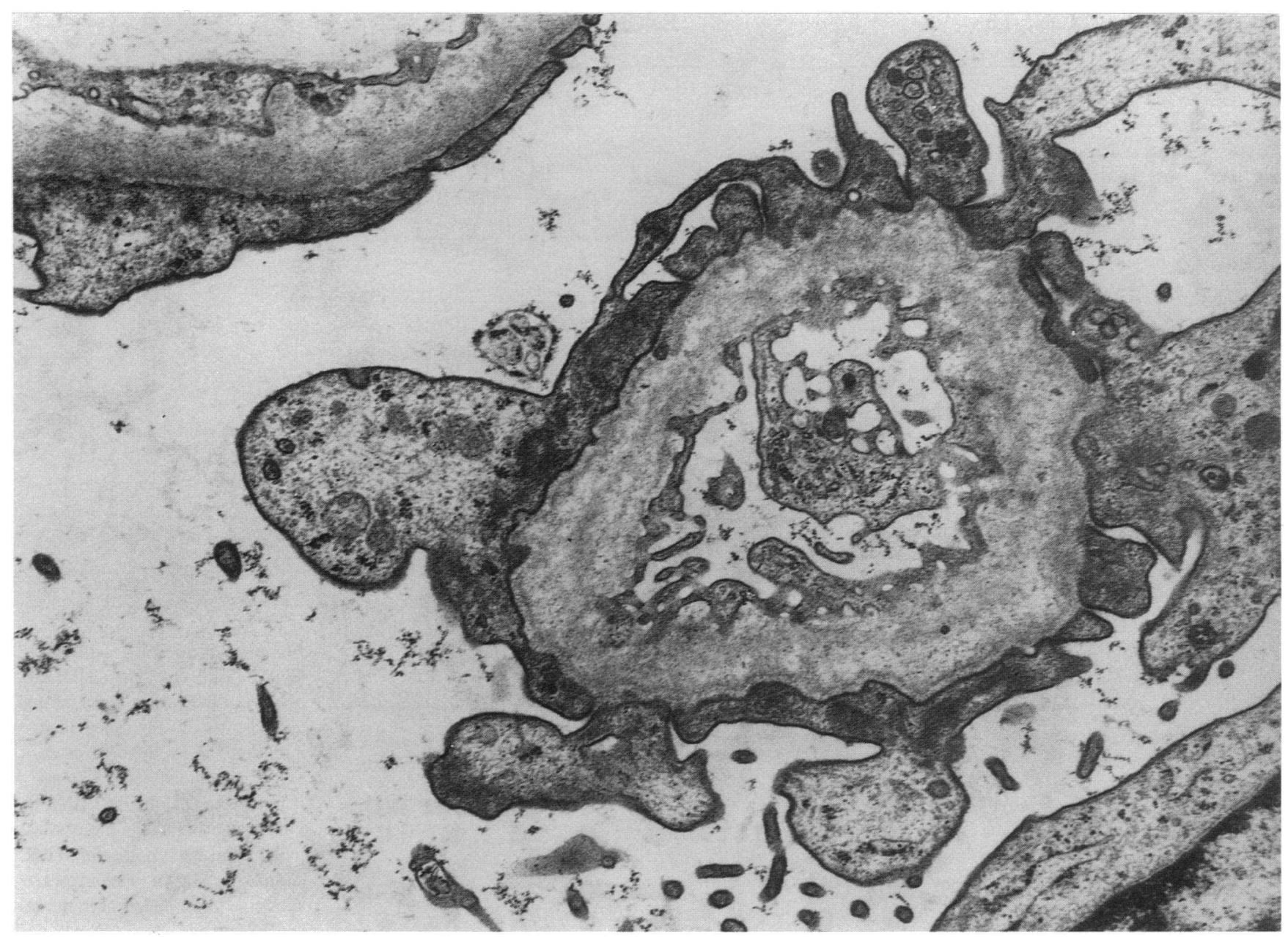

Figure 1. Electron micrograph of the kidney biopsy. The picture shows a capillary with a thickened, irregular, and split basement membrane, characteristic of Alport disease. $\times 23,000$.

sequence (8). PCR was performed in $100 \mu \mathrm{l}$ of $50 \mathrm{mM} \mathrm{KCl}, 10 \mathrm{mM}$ Tris-HCl, pH 8.3, $1.5 \mathrm{mM} \mathrm{MgCl}, 200 \mu \mathrm{M}$ dNTPs, $0.01 \%$ gelatin, 2.5 U Taq DNA polymerase, $1 \mu \mathrm{g}$ genomic DNA, and $25 \mathrm{pM}$ of each primer. A 4-min denaturation step at $95^{\circ} \mathrm{C}$ was followed by 30 cycles of amplification with $30 \mathrm{~s}$ at $54^{\circ} \mathrm{C}$ (exon 15) or at $62^{\circ} \mathrm{C}$ (exon 46 ), 30 $\mathrm{s}$ at $72^{\circ} \mathrm{C}$, and $30 \mathrm{~s}$ at $95^{\circ} \mathrm{C}$. The amplified fragments, 97 and $150 \mathrm{bp}$ in length, respectively, were purified, ligated in SmaI-cut pGEM-3Z vector, and transformed into XL1-blue cells by electroporation. 22 insert-containing clones of exon 15 and 31 clones of exon 46 were isolated and sequenced as described previously (6).

For the quantitative analysis by dot blots, the amplified fragments were purified using the QIAquick-spin PCR Purification Kit (QIAGEN, Hilden, Germany), denatured at $95^{\circ} \mathrm{C}$ for $10 \mathrm{~min}$, chilled on ice, transferred to a Hybond- $\mathrm{N}^{+}$membrane (Amersham International, Buckinghamshire, United Kingdom) and fixed by exposure to ultraviolet light. A DNA sample of a normal female and a plasmid containing the mutant exons were used as controls. To measure the amount of amplified DNA on the filters, they were first hybridized with the appropriate ${ }^{32} \mathrm{P}$-labeled forward primer in $6 \times$ SSC, $5 \times$ Denhardt's, $0.5 \%$ SDS overnight, washed for $10 \mathrm{~min}$ at $58^{\circ} \mathrm{C}$ (exon 15 ) or $62^{\circ} \mathrm{C}$ (exon 46) in $2 \times$ SSPE, $0.1 \%$ SDS, and autoradiographed at $-70^{\circ} \mathrm{C}$. The filters were then stripped with $0.4 \mathrm{~N} \mathrm{NaOH}$ at $60^{\circ} \mathrm{C}$ for $30 \mathrm{~min}$ and hybridized with allele-specific oligonucleotides (exon 15 normal sequence: TGAGAAGGGTGAGCAAGG, mutant: TGAGAAGGTTGAGCAAGG; exon 46 normal sequence: CAGGAGGGCGCAAAGGAG, mutant: CAGGAGGGTGCAAAGGAG). Quantification was performed on a laser densitometer (Molecular Dynamics, Inc., Sunnyvale, CA).

The common promoter of COLAA5 and COL4A6 was sequenced as follows. A fragment containing the common promoter region was amplified from genomic DNA isolated from the patient's kidney and a normal control. Two primers (F: 5'-CCAAAGACTCAGGGCCAGTAAGAA, R: 5'-AACTCTCTGTTCTGTGAGCAGCTG) were selected from the exon 1 sequence of, respectively, COL4A5 and COL4A6 (1). PCR was performed as described above with 25 cycles of $40 \mathrm{~s}$ at $94^{\circ} \mathrm{C}, 30 \mathrm{~s}$ at $60^{\circ} \mathrm{C}$, and $40 \mathrm{~s}$ at $72^{\circ} \mathrm{C}$. An amplified fragment of $766 \mathrm{bp}$ was cloned and sequenced as described above.

The X-inactivation pattern of the patient was investigated by Southern blotting with the pSPT/PGK probe for the PGK1 gene exactly as described (9). The filter was autoradiographed at $-70^{\circ} \mathrm{C}$ and analyzed on a laser densitometer (Molecular Dynamics, Inc.).

\section{Results}

Morphological investigation. The light microscopic lesions were mild. In one out of nine glomeruli a segmental adhesion was found. Some tubular atrophy and one obsolete glomerulus were found. In one tubulus red blood cells were present.

On electron microscopy the main lesion was located in the capillary wall and consisted of an irregular thinning and thickening of the basement membrane. The outer contour of the basement membrane was wavy in many capillaries. The lamina densa was disintegrated into small fragments. In the thick parts of the basement membrane, electron dense inclusions were found (Fig. 1). 


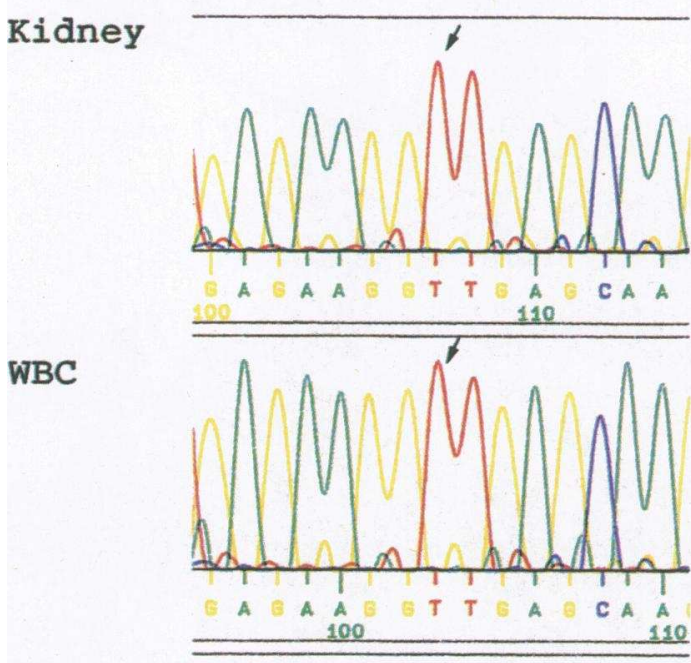

NORMAL

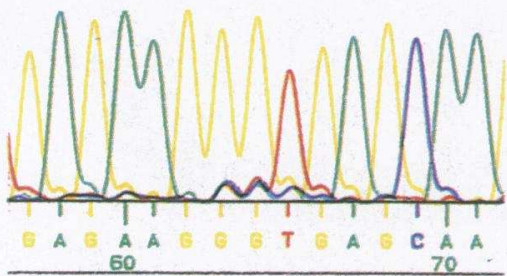

EXON 46
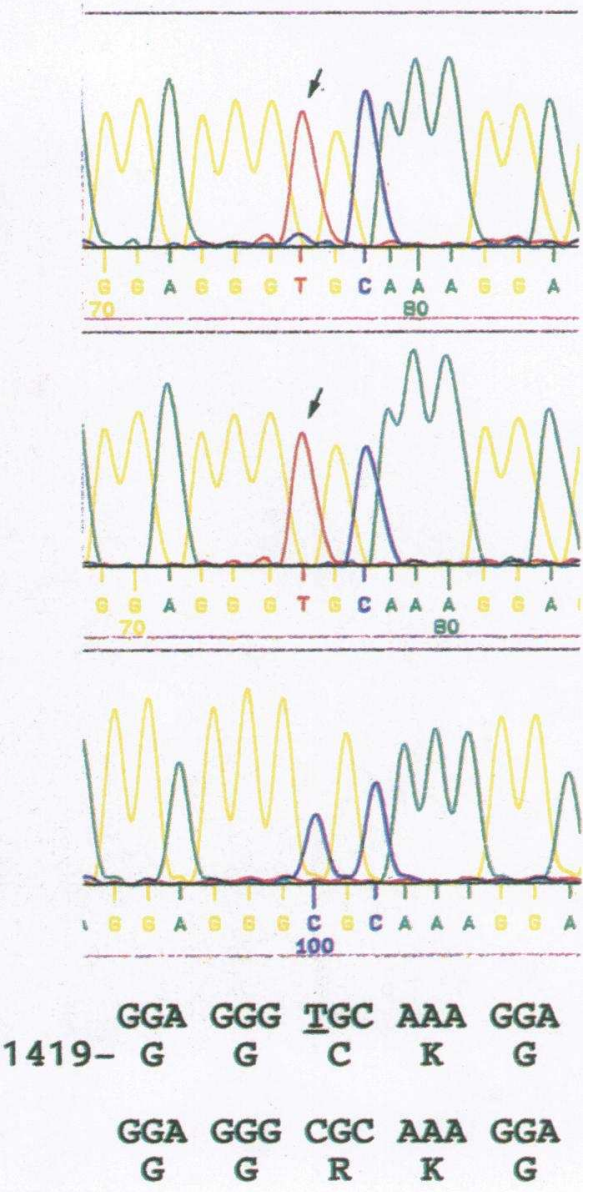

Figure 2. Sequencing of the COL4A5 mRNA. The COL4A5 mRNA of the patient and a normal control was reverse transcribed, amplified, and sequenced as described previously (6). The sequencing traces obtained from a kidney and WBC sample of the patient and from a normal WBC control are shown. The arrows show the point mutation in the respective exons.
Detection of two missense mutations. Sequencing of the COL4A5 cDNA isolated from a kidney biopsy sample and from WBC of the patient revealed two point mutations. The first mutation, G289V, occurred in exon 15 and converted a GGT codon (Gly) to a GTT codon ( Val), while the second mutation, R1421C, was located in exon 46 and changed a CGC codon (Arg) to a TGC codon (Cys) (Fig. 2, numbering of all amino acids according to Zhou et al. [10]). Of interest, the sequencing trace for both the kidney sample and the WBC sample did not show the normal sequence, suggesting that both mutations occurred in the same COL4A5 allele and that the normal allele was either not present or not expressed. Identical results were obtained after sequencing the products of two other independent reverse transcription-PCR experiments.

To investigate this the corresponding exons of the COL4A5 gene were amplified by PCR from genomic DNA of the patient, cloned, and sequenced. Among 22 clones isolated for exon 15, 10 copies showed the normal sequence and 12 copies showed the G289V mutation, while of 31 clones derived from exon 46, 10 showed the normal sequence and 21 showed the R1421C mutation (results not shown). These results confirmed the presence of the mutations at the genomic level and showed that the patient was heterozygous for both mutations. In addition a cytogenetic analysis of $20 \mathrm{G}$-banded metaphase spreads from WBC of the patient showed a 46,XX karyotype with two morphologically normal $\mathrm{X}$ chromosomes.

Since the occurrence of two point mutations in the same patient must be a rare event, their eventual presence in the normal population was investigated. Both exon 15 and 42 were amplified from 46 normal female DNA samples and a dot blot analysis was performed with allele-specific oligonucleotides for the normal and mutant form of each exon. In all samples, only the normal sequence was detected (results not shown) arguing against the possibility that one of the mutations was in fact a polymorphism.

Further evidence that both mutations occurred on the same chromosome was obtained upon analysis of EBV-transformed cells generated from the patient. Karyotyping showed that $80 \%$ of the EBV-transformed cells had a 45,XO karyotype and 20\% had a normal 46,XX karyotype. Both exon 15 and 46 were amplified from genomic DNA isolated from kidney, WBC, and the EBV-transformed cells of the patient. The PCR products were purified and blotted onto nylon membranes and the amount of DNA on the membranes was quantitated by hybridization to one of the PCR primers (Fig. 3, Table I). The membranes were then hybridized to allele-specific oligonucleotides detecting, respectively, the normal or the mutant sequence. The corresponding signals were scanned with a densitometer and normalized for the amount of DNA present on the membrane. Fig. 3 and Table I show that, for both exons, the signals obtained for the normal and the mutant sequence were nearly equal for the genomic DNA isolated from kidney and WBC of the patient. The signals obtained for the normal allele with kidney and WBC DNA were approximately half as strong as the signals obtained 


\section{$\begin{array}{llllllllll}A & B & C & D & E & A & B & C & D & E\end{array}$}

\section{PROBE}

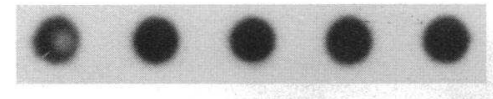

Normal
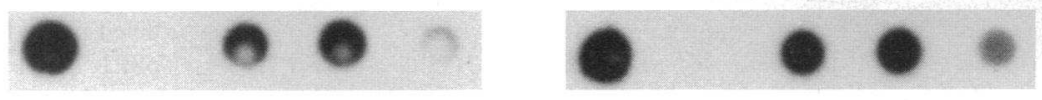

Figure 3. Dot blot analysis of the missense mutations. Exons 15 and 46 were amplified from genomic DNA of a normal female control $(A)$, kidney $(C)$, WBC $(D)$, and EBV-transformed cells $(E)$ of the patient and from a plasmid containing the mutant sequence $(B)$. The PCR products were blotted onto a nylon membrane, then hy-
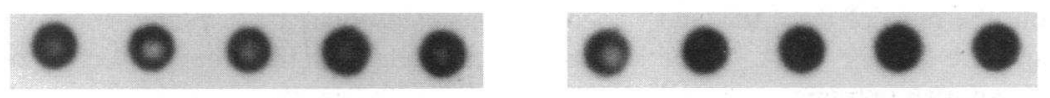
bridized with the forward PCR primer $(F P)$ to quantify the amount of DNA present on the membrane, stripped, and rehybridized with oligonucleotides Mutant
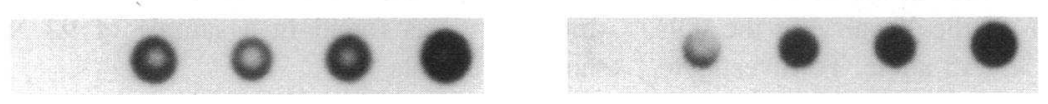
specific for either the normal or the mutant sequence.

for a normal female genomic DNA control. However, with DNA isolated from the EBV-transformed cells of the patient, the ratio of the normalized signals for the normal and the mutant sequence was approximately 1:9 for both exons showing that both mutations were present on the same chromosome and strongly suggesting a clonal origin of the $45, \mathrm{XO}$ cells in the EBV-transformed population.

Genomic analysis of the COLAA5 gene and promoter. To investigate the exclusive expression of the mutant allele, first the genomic structure of the normal and the mutant allele was analyzed. An equal amount of genomic DNA from the patient's kidney, a female and a male WBC control was digested with EcoRI and used for Southern blotting with cDNA probes spanning the complete COL4A5 gene. All probes detected only fragments of the expected size. In addition, the intensity of the signal obtained with DNA of the patient was equal to the signal observed with the female control and approximately twice the signal observed for the male sample (results not shown). This

Table I. Dot Blot Analysis of the Missense Mutations

\begin{tabular}{lcccc}
\hline & $\begin{array}{c}\text { Female } \\
\text { control }\end{array}$ & Kidney & WBC & EBV cells \\
\hline Exon 15 & & & & \\
$\quad$ Probe & & & & \\
$\quad$ Normal & 1.93 & 0.90 & 1.00 & 0.06 \\
$\quad$ Mutant & - & 0.90 & 1.00 & 2.12 \\
Exon 46 & & & & \\
Probe & & & & \\
$\quad$ Normal & 3.20 & 1.10 & 1.00 & 0.21 \\
Mutant & - & 0.90 & 1.00 & 1.52 \\
\hline
\end{tabular}

The autoradiographs of Fig. 3 were analyzed with a laser densitometer (Molecular Dynamics, Inc.). The absorbance of the dots for the WBC DNA was set to 1 and all other measurements were normalized accordingly. excluded a major rearrangement of the COL4A5 genes of the patient.

Next the possibility that a mutation occurred in the promoter of the normal COL4A5 allele was investigated. The COL4A5 gene maps to chromosome Xq22 head-to-head to the COL4A6 gene, separated by a common promoter (1) in an arrangement similar to the COL4A1 and the COL4A2 genes (11). Two primers were selected from exon 1 of, respectively, COL4A5 and COL4A6, and PCR conditions were optimized to amplify the common promoter. The promoter was amplified from genomic kidney DNA from the patient and from a normal control. Six independent clones from the patient were sequenced and showed exactly the same sequence as the control sample (see Methods).

$X$-inactivation pattern. To study the $\mathrm{X}$-inactivation pattern of the patient a BstX1 polymorphism of the PGK1 gene was analyzed by Southern hybridization. After digestion of genomic DNA with BstXI and PstI, the pSPT/PGK probe (see Methods) can detect a BstXI polymorphism generating signals of, respectively, 1.05 and $0.9 \mathrm{~kb}$. These fragments contain eight HpaII sites which are methylated on the inactive chromosome and nonmethylated on the active $\mathrm{X}$ chromosome. Upon digestion with the methylation-sensitive restriction enzyme HpaII, the 1.05- or $0.9-\mathrm{kb}$ fragments derived from the active $\mathrm{X}$ chromosome will specifically disappear. Genomic DNA of kidney, WBC, and the EBV-transformed cells of the patient was digested with BstXI and PstI. Half of the samples were then digested with HpalI. All samples were separated on an agarose gel, blotted onto a nylon membrane, and hybridized with the pSPT/PGK probe. Genomic WBC DNA from a normal female was used as a control. The results are shown in Fig. 4 and Table II. The BstXI/PstI digests of WBC and kidney DNA showed the patient to be heterozygous with equal signals for the 1.05 and the 0.9-kb allele, further illustrating the presence of two $\mathrm{X}$ chromosomes in the kidney cells. With the genomic DNA isolated from the EBV-transformed cells, the signal for the 1.05$\mathrm{kb}$ allele was much stronger than the signal for the $0.9-\mathrm{kb}$ allele. This corresponded very well with the karyotypic result for the 


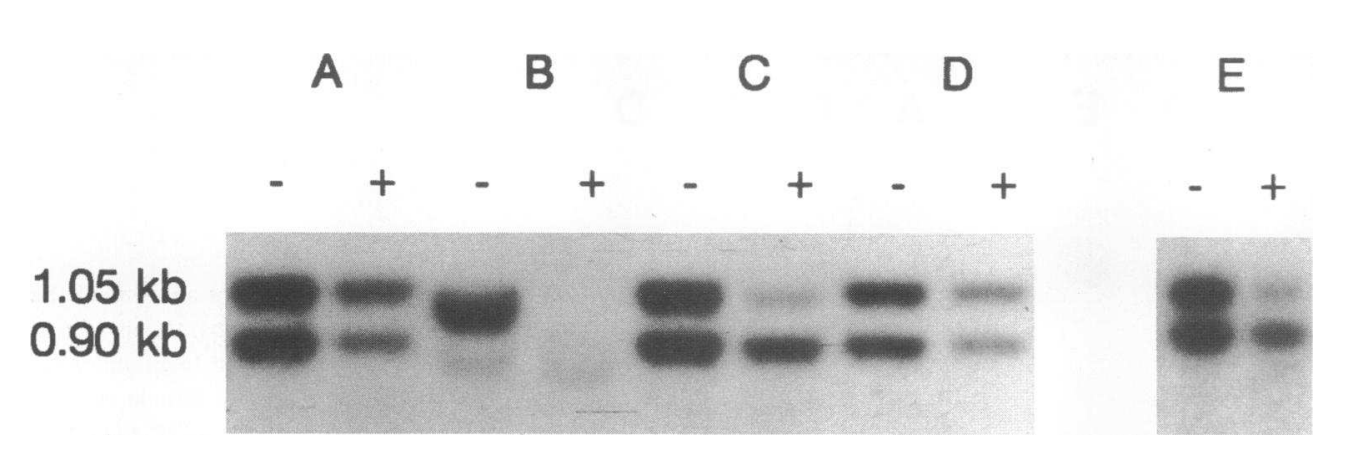

Figure 4. X-inactivation analysis. Genomic DNA from two normal female WBC controls $(A$ and $D)$, and kidney $(C)$, EBV-trans-

formed cells $(B)$, and WBC $(E)$ of the patient were digested with BstXI and PstI. Half of each sample was then digested with HpaII. All samples were sized on an agarose gel, blotted on a nylon membrane, and hybridized with a labeled probe for the PGK1 gene: pSPT/PGK.

cells. This also indicated that the mutant COL4A5 allele must be associated with the 1.05-kb PGK1 allele on the same X chromosome. After HpalI digestion, the ratio of the 1.05- and the 0.9-kb allele for the WBC and the kidney DNA of the patient was 1:9 (Table II) demonstrating an extremely skewed inactivation pattern of the $\mathrm{X}$ chromosome in the kidney with a preferential inactivation of $\sim 91 \%$ of the $X$ carrying the 0.9 kb PGK1 allele and the normal COL4A5 allele. To investigate whether this skewed inactivation pattern was specific for kidney tissue, the experiment was repeated with DNA isolated from WBC of the patient. Fig. 4, lane $E$, shows that a similarly skewed $\mathrm{X}$ inactivation occurred in this tissue. The $1.05-\mathrm{kb}$ allele of the EBV-transformed cells disappeared almost completely as expected, since the $45, \mathrm{XO}$ cells must have an active $\mathrm{X}$ chromosome to survive.

\section{Discussion}

In this patient the clinical data, the light microscopy, and the electron microscopy were characteristic for Alport's disease. Indeed, two missense mutations were detected in the COL4A5 mRNA of the female patient and their presence in genomic DNA was confirmed. The first mutation replaced a Gly residue of the Gly-Xaa-Yaa motif in a collagenous domain of COL4A5 by a more bulky Val residue. Because of their small size, the Gly residues are essential for the formation of the triple helical structure of the collagenous domains of the collagen monomers. Several Gly substitutions in the collagenous domains of COL4A5 were reported in Alport patients $(10,12,13)$, strongly suggesting that this mutation was sufficient to explain the Alport pathology of the patient. However, a second mutation was found substituting a Cys residue for an Arg at position 1421 proximal to the COOH-terminal $\mathrm{NC1}$ domain of COL4A5. The NC1 domain of COL4A5 contains 12 Cys residues, 8 of which play a direct role in the formation of intermolecular bonds of the collagen dimers (14). It is therefore conceivable that the presence of an additional Cys residue in proximity of the $\mathrm{NCl}$ domain interferes with the proper assembly of the supramolecular type IV collagen structure. Unfortunately, no samples of other family members were available, but neither one of the mutations was found in a sample of $92 \mathrm{X}$ chromosomes of normal controls.

Two independent observations showed that both mutations were present in the same COL4A5 gene. The sequencing traces showed only the presence of the mutant sequence for both mutations, indicating that the normal sequence could only be present in $<10 \%$ of the sequenced PCR products. The selective amplification of the mutant sequence was highly unlikely. Both mutations occurred in cDNA fragments obtained by independent amplification reactions, only one basepair was changed in each case and both the mutant and the normal sequence were amplified for both exons from genomic DNA with a similar efficiency as shown by the dot blot experiments. On the other hand, when the dot blot experiments were performed with genomic DNA isolated from the EBV-transformed cells of the patient, 80\% of the PCR products carried the mutant sequence for both exons, which corresponded very well with the fact that $80 \%$ of the EBV cells had a 45,XO karyotype.

The exclusive expression of the mutant allele in the kidney, and hence the severe Alport phenotype of the female patient, could be explained in several ways. First, the patient could be a mosaic for the $46, \mathrm{XX}$ and the $45, \mathrm{XO}$ karyotype. It was reported that $\sim 15 \%$ of the Turner patients are $46, \mathrm{XX} / 45$, XO mosaics (15), and the occurrence of $80 \%$ of the cells with a 45,XO karyotype in the EBV-transformed cells from the patient could point in this direction. However, the patient showed no clinical manifestation of Turner syndrome, and in the $20 \mathrm{WBC}$ metaphase spreads which were analyzed a 46,XX karyotype

Table II. X-Inactivation Analysis

\begin{tabular}{|c|c|c|c|c|c|c|c|c|}
\hline \multirow[b]{2}{*}{ Hpall } & \multicolumn{2}{|c|}{ Control 1} & \multicolumn{2}{|c|}{ EBV cells } & \multicolumn{2}{|c|}{ Kidney } & \multicolumn{2}{|c|}{ Control 2} \\
\hline & - & + & - & + & - & + & - & + \\
\hline \multicolumn{9}{|l|}{ Allele } \\
\hline $1.05 \mathrm{~kb}$ & 5070 & 1480 & 4008 & 0 & 4877 & 215 & 2150 & 519 \\
\hline $0.90 \mathrm{~kb}$ & 4094 & 831 & 171 & 110 & 4385 & 1992 & 1860 & 516 \\
\hline Ratio & \multicolumn{2}{|c|}{$58: 42$} & \multicolumn{2}{|c|}{$0: 100$} & \multicolumn{2}{|c|}{ 9:91 } & \multicolumn{2}{|c|}{$47: 53$} \\
\hline
\end{tabular}

The autoradiographs of Fig. 4 were analyzed on a laser densitometer. Absorbances are given in arbitrary units. The signals obtained after HpaII digestion were normalized with values obtained for either allele without HpaII digestion before calculating the ratio of the signal for both alleles. 
was observed. More importantly, dot blot analysis showed that for both exon 15 and 46 the mutant and the normal sequence were present in equal amounts in the DNA of the kidney and WBC of the patient. In addition, a normal dosage for the COL4A5 and the PGK1 genes was observed on Southern blots which compared kidney DNA from the patient to normal female and male controls. Taken together these results indicate that the vast majority of the WBC and the kidney cells of the patient must have a normal $46, \mathrm{XX}$ karyotype. The generation of the EBV-transformed cells of which $90 \%$ show a $45, \mathrm{XO}$ karyotype must therefore be the result of a clonal expansion of a 45,XO cell or more unlikely a tissue culture artifact. Second, a mutation in the second COL4A5 gene of the patient could prevent its expression or modify dramatically the stability of its mRNA. Southern analysis of the COL4A5 genes did not detect any major deletions or rearrangements of the COL4A5 genes of the patient. Sequencing of the common promoter region between the COL4A5 and the COL4A6 genes also did not detect any mutation. However, we could not exclude mutations which would affect enhancer sequences located elsewhere in the COL4A5 or COL4A6 gene. This would imply that the patient is a compound heterozygote for the Alport mutation which must be an extremely rare event.

A skewed X-inactivation pattern has been shown to be responsible for the phenotypic expression of diseases in women carriers of $X$-linked disease genes $(16,17)$ and could also be responsible for the observed absence of expression of the normal allele of COL4A5 in the patient. A study of the methylation of the 5' end of the PGK1 gene showed a clearly skewed pattern of $X$ inactivation. In the kidney and WBC of the patient $\sim 90 \%$ of the $\mathrm{X}$ chromosomes carrying the normal COL4A5 allele were inactivated, which most probably explains the absence of detectable normal COL4A5 mRNA in both WBC and kidney of this patient. The inactivation of the $\mathrm{X}$ chromosome is normally a random event occurring early during embryogenesis, and the extremely skewed pattern of $\mathrm{X}$ inactivation observed here is occasionally expected from a normally distributed process. Similar ratios for $\mathrm{X}$ inactivation have indeed been described in normal women. This would then imply that there is no selection against cells carrying the COL4A5 mutation during embryogenesis and is in agreement with the absence of a correlation found between $\mathrm{X}$ inactivation and the $\mathrm{X}$ chromosome carrying the AS gene in 43 women reported by Vetrie et al. (5). The same study also failed to detect a correlation between the inactivation pattern and the severity of the Alport pathology in these females. It should be noted that in the study by Vetrie et al. (5) the Xinactivation pattern was studied in WBC, which do not necessarily reflect the X-inactivation pattern in the affected tissues (18), although in this study no difference in the $\mathrm{X}$-inactivation pattern in WBC and kidney of the patient was observed. The nonrandom pattern of $\mathrm{X}$ inactivation observed in the patient reported here could also be the expression of a genetic variant at the XIC locus or a major anomaly of the normal $\mathrm{X}$, but in the absence of samples of relatives of the patient this hypothesis cannot be tested.

In conclusion, we have detected two missense mutations in the same COL4A5 gene of a female Alport patient. The severe Alport phenotype of the patient correlated with the absence of detectable amounts of COL4A5 mRNA with the normal sequence in the kidney and WBC. It is suggested that this is the result of the extremely skewed pattern of $\mathrm{X}$ inactivation, with $\sim 90 \%$ of the $X$ carrying the normal COL4A5 allele being inactivated.

\section{Acknowledgments}

This work was supported by the Interuniversity Network for Fundamental Research sponsored by the Belgian Government (1991-1996). These investigations have been supported by grant 3.9003 .92 of the Nationaal Fonds voor Wetenschappelijk Onderzoek of Belgium. Peter Marynen is a Bevoegdverklaard Navorser of the Nationaal Fonds voor Wetenschappelijk Onderzoek, Belgium.

\section{References}

1. Zhou, J., T. Mochizuki, H. Smeets, C. Antignac, P. Laurila, A. de Paepe, K. Tryggvason, and S. T. Reeders. 1993. Deletion of the paired $\alpha 5$ (IV) and $\alpha 6$ (IV) collagen genes in inherited smooth muscle tumors. Science (Wash. DC). 261:1167-1169.

2. Grünfeld, J. P., L. H. Noël, S. Hafez, and D. Droz. 1985. Renal prognosis in women with hereditary nephritis. Clin. Nephrol. 23:267-271.

3. Hudson, B. G., S. T. Reeders, and K. Tryggvason. 1993. Type IV collagen structure, gene organization and role in human diseases. J. Biol. Chem. 268:26033-26036.

4. Tryggvason, K., J. Zhou, S. L. Hostikka, and T. B. Show. 1993. Molecular genetics of Alport syndrome. Kidney Int. 43:38-44.

5. Vetrie, D., F. Flinter, M. Bobrow, and A. Harris. 1992. X inactivation patterns in females with Alport's syndrome: a means of selecting against a deleterious gene? J. Med. Genet. 29:663-666.

6. Guo, C., B. Van Damme, R. Van Damme-Lombaerts, H. Van den Berghe, J. J. Cassiman, and P. Marynen. 1993. Differential splicing of COL4A5 mRNA in kidney and white blood cells. A complex mutation in the COL4A5 gene of an Alport patient deletes the NCl domain. Kidney Int. 44:1316-1321.

7. Müllenbach, R., P. J. L. Lagoda, and C. Welter. 1989. An efficient saltchloroform extraction of DNA from blood and tissues. Trends Genet. 5:391.

8. Zhou, J., S. L. Hostikka, L. T. Chow, and K. Tryggvason. 1991. Characterisation of the $3^{\prime}$ half of the human type IV collagen $\alpha 5$ gene that is affected in the Alport syndrome. Genomics. 9:1-9.

9. Vogelstein, B., E. R. Fearon, S. R. Hamilton, A. C. Preisinger, H. F. Willard, A. M. Michelson, A. D. Riggs, and S. H. Orkin. 1987. Clonal analysis using recombinant DNA probes from the X-chromosome. Cancer Res. 47:48064813.

10. Zhou, J., J. M. Herz, A. Leinonen, and K. Tryggvason. 1992. Complete amino acid sequence of the human $\alpha 5$ collagen chain and identification of a single base mutation in exon 29 from the $3^{\prime}$ end converting glycine-521 in the collagenous domain to cysteine in an Alport syndrome patient. J. Biol. Chem. 267:1247512481 .

11. Soininen, R., M. Huotari, S. L. Hostikka, D. J. Prockop, and K. Tryggvason. 1988. The structural genes for $\alpha 1$ and $\alpha 2$ chains of human type IV collagen are divergently encoded on opposite DNA strands and have an overlapping promoter region. J. Biol. Chem. 263:17217-17220.

12. Zhou, J., J. M. Hertz, and K. Tryggvason. 1992. Mutation in the $\alpha 5$ (IV) collagen chain in juvenile-onset Alport syndrome without hearing loss or ocular lesions: detection by denaturing gradient gel electrophoresis of a PCR product. Am. J. Hum. Genet. 50:1291-1300.

13. Knebelmann, B., G. Deschenes, F. Gros, M. C. Hors, J. P. Grünfeld, K. Tryggvason, M. C. Gubler, and C. Antignac. 1992. Substitution of arginine for glycine 325 in the collagen $\alpha 5$ (IV) chain associated X-linked Alport syndrome: characterization of the mutation by direct sequencing of PCR-amplified lymphoblast cDNA fragments. Am. J. Hum. Genet. 51:135-142.

14. Siebold, B., R. Deutzmann, and K. Kühn. 1988. The arrangement of intra- and intermolecular disulfide bonds in the carboxyterminal, non collagenous aggregation and cross-linking domain of basement-membrane type IV collagen. Eur. J. Biochem. 176:617-624.

15. Hook, E. B., and D. Warburton. 1983. The distribution of chromosomal genotypes associated with Turner's syndrome: livebirth prevalence rates and evidence for diminished fetal mortality and severity in genotypes associated with structural X abnormalities or mosaicism. Hum. Genet. 64:24-27.

16. Brown, R. M., and G. K. Brown. 1993. X chromosome inactivation and the diagnosis of X linked disease in females. J. Med. Genet. 30:177-184.

17. Jorgensen, A. L., J. Philip, W. H. Raskind, M. Matsushita, V. Dreyer, and A. G. Motulsky. 1992. Different patterns of X inactivation in MZ twins discordant for red-green color-vision deficiency. Am. J. Hum. Genet. 51:291-298.

18. Gale, R. E., H. Wheadon, P. Boulos, and D. C. Linch. 1994. Tissue specificity of X-chromosome inactivation patterns. Blood. 83:2899-2905. 